\title{
Comparison of the clinical efficacy between single-agent and dual-agent concurrent chemoradiotherapy in the treatment of unresectable esophageal squamous cell carcinoma: a multicenter retrospective analysis
}

Jie Li ${ }^{1}$, Youling Gong ${ }^{2}$, Peng Diao ${ }^{3}$, Qingmei Huang ${ }^{1,4}$, Yixue Wen ${ }^{1,4}$, Binwei Lin ${ }^{4}$, Hongwei Cai ${ }^{5}$, Honggang Tian ${ }^{6}$, Bing $\mathrm{He}^{7}$, Lanlan $\mathrm{Ji}^{8}$, Ping $\mathrm{Guo}^{9}$, Jidong Miao ${ }^{10}$ and Xiaobo Du${ }^{1 *}$

\begin{abstract}
Background: Some Chinese patients with esophageal squamous cell carcinomaare often treated with single-agent concurrent chemoradiotherapy. However, no results have been reported from randomized controlled clinical trials comparing single-agent with double-agent concurrent chemoradiotherapy. It therefore remains unclear whether these regimens are equally clinically effective. In this study, we retrospectively analyzed and compared the therapeutic effects of single-agent and double-agent concurrent chemoradiotherapy in patients with unresectable esophageal squamous cell carcinoma.

Methods: This study enrolled 168 patients who received definitive concurrent chemoradiotherapy for locally advanced unresectable esophageal squamous carcinoma at 10 hospitals between 2010 and 2015. We evaluated survival time and toxicity. The Kaplan-Meier method was used to estimate survival data. The log-rank test was used in univariate analysis A Cox proportional hazards regression model was used to conduct a multivariate analysis of the effects of prognostic factors on survival.
\end{abstract}

Results: In this study, 100 (59.5\%) and 68 patients (40.5\%) received single-agent and dual-agent combination chemoradiotherapy, respectively. The estimate 5 -year progression-free survival (PFS) rate and overall survival (OS) rate of dual-agent therapy was higher than that of single-agent therapy $(52.5 \%$ and $40.9 \%, 78.2 \%$ and $60.7 \%$, respectively), but there were no significant differences ( $P=0.367$ and 0.161 , respectively). Multivariate analysis showed that sex, age,and radiotherapy dose had no significant effects on OS or PFS. Only disease stage was associated with OS and PFS in the multivariable analysis ( $P=0.006$ and 0.003 , respectively). In dual-agent group, the incidence of acute toxicity and the incidence of 3 and 4 grade toxicity were higher than single-agent group. (Continued on next page)

\footnotetext{
*Correspondence: duxiaobo2005@126.com

${ }^{1}$ Department of Oncology, Mian Yang Central Hospital, Mianyang 621000,

People's Republic of China

Full list of author information is available at the end of the article
} 
(Continued from previous page)

Conclusion: The 5-year PFS and OS rates of dual-agent therapy were higher than those of single-agent concurrent chemoradiotherapy for patients with unresectable esophageal squamous cell carcinoma; however, there were no significant differences in univariate analysis and multivariable analysis. Single-agent concurrent chemotherapy had less toxicity than a double-drug regimen. Therefore, we suggest that single therapis not inferior to dual therapy $y$. In the future, we aim to confirm our hypothesis through a prospective randomized study.

Keywords: Sophageal squamous cell carcinoma, Concurrent chemoradiotherapy, Single-agent chemotherapy, Dual-agent chemotherapy,

\section{Background}

Esophageal cancer is the sixth most common cause of cancer-related deaths and the eighth most common cancer worldwide. Current estimates suggest that approximately 500,000 new esophageal cancer cases are diagnosed and more than 400,000 related deaths occur annually worldwide. In addition, the incidence of esophageal cancer continues to increase in contrast to decreases in the incidence of many other cancers. China has a high incidence of esophageal cancer. Squamous cell carcinoma (SCC) is the most common histological type of esophageal cancer, accounting for more than $90 \%$ of cases in China (according to data from 2012) and approximately $53 \%$ of cases worldwide [1-3].

Surgery is considered the most important treatment modality for patients with esophageal cancer. However, $40-60 \%$ of patients are deemed ineligible for surgery at the initial diagnosis [4]. For patients with locally advanced, unresectable esophageal carcinoma, the standard treatment comprises radiation and concurrent chemotherapy. A landmark randomized trial conducted in 1999 by the Radiation Therapy and Oncology Group (RTOG)85-01 reported 2-year overall survival (OS) rates of $10 \%$ and $36 \%$ for radiotherapy alone vs. concurrent chemoradiotherapy with 5-fluorouracil/cisplatin, respectively, and corresponding 5-year OS rates of $0 \%$ and $26 \%$, respectively [5]. Consequently, radiation with concurrent chemotherapy has become a widely accepted standard treatment regimen for patients with locally advanced, inoperable esophageal carcinoma.

Standard protocols for concurrent chemoradiotherapy generally involve platinum agent-based or fluorouracilbased combination chemotherapies. However, the current trend of an aging population has resulted in increased numbers of elderly patients with esophageal cancer in China. Some of these older patients with locally advanced unresectable SCC, as well as those with reduced food intake, organ dysfunction, and/or chronic comorbidities, cannot tolerate the toxic effects of dual-agent concurrent chemoradiotherapy. Accordingly, Chinese patients are often treated with single-agent concurrent chemoradiotherapy. However, no results have been reported from randomized controlled clinical trials comparing single-agent with double-agent concurrent chemoradiotherapy. It therefore remains unclear whether these regimens are equally clinically effective. In this study, we retrospectively analyzed and compared the therapeutic effects of single-agent and double-agent concurrent chemoradiotherapy in patients with unresectable esophageal SCC.

\section{Methods \\ Patient selection}

The study was approved by the Medical Ethics Committee of Mianyang Central Hospital (approval no. S2016055). As clinical data were analyzed anonymously, ethics committee agrees that we were not required to obtain informed consent from the patients. The study was performed from October 2016 to February 2017 in 10 hospitals in China. We retrospectively reviewed patients with non-metastatic esophageal cancer at 10 hospitals in China between January 2010 and December 2015. Eligible patients had been pathologically confirmed to have SCC and received esophagoscope examination and/or esophagus barium meal, CT of chest, neck or upper abdomen,and Whole body bone scan. Disease stages of I-III were determined using China'sclinical staging criteria for the non-operative treatment of esophageal cancer [6]. Patients who were treated with definitive concurrent chemoradiotherapy were included in the study. Patients who underwent surgery, received irradiation doses $<50 \mathrm{~Gy}$, received palliative care, had unclear staging or non-SCC, received tri-modal chemotherapy without any follow up informationwere excluded. One hundred and 68 patients were enrolled in the study. The patients' clinical records were reviewed from the time of diagnosis until death or the last follow-up, whichever came first. We recorded information related to the age at diagnosis, sex, tumor $(\mathrm{T})$ and nodal $(\mathrm{N})$ stages, location of tumor, ECOG score, radiation therapy dose, and chemotherapy regimen. All patients, except for those clearly identified as deceased in the records, were followed up via telephone or clinical visits. The followup deadline was set to January 20, 2017. The median follow-up time was 24 months (rage 1-60 months). 


\section{Treatment}

According to patient age, ECOG score, nutritional status, among other parameters, the physician prepares the treatment regimen plan, which is then discussed with the patients and/or family members to determine the treatment regimen. All patients underwent intensity modulated radiotherapy; 4 patients additionally received three-dimensional conformal radiotherapy (range: 5070 Gy/25-35 fractions). Concurrent chemotherapy agents included 5-fluorouracil or the oral agents S1 and capecitabine, cisplatin, carboplatin, oxaliplatin, paclitaxel, and docetaxel. Single-agent and dual-agent regimens comprised weekly and 3-week cycles, respectively. Patients were categorized into different treatment arms based on treatment with single-agent or double-agent chemoradiation. Toxicity was recorded according to the National Cancer Institute Common Toxicity Criteria (version 3.0). For this study, acute toxicities were defined as those occur $\leq 90$ days from the start of radiotherapy.

\section{Statistical methods}

OS was defined as the time from treatment initiation until patient death. PFS was defined as the time from treatment initiation until the first objective tumor progression or death for any cause. Objective tumor progression was determined by biopsy and/or CT,PET/CT, Whole body bone scan, or MRI. SPSS 22.0 software (SPSS, Inc., Chicago, IL, USA) was used for the statistical analysis. Student's t-test was used for comparison of means. Fisher's exact test. Was used for comparisons of categorical data. The Kaplan-Meier method was used to estimate survival data, and differences between curves were analyzed using the log-rank test. A Cox proportional hazards regression model was used to conduct a multivariate analysis of the effects of prognostic factors on survival. All statistical tests were conducted at the 5\% level, and 95\% confidence intervals $(\mathrm{CI})$ were calculated. A bilateral $P$ value $<0.05$ indicated a statistically significant difference.

\section{Results}

We identified 168 patients treated with concurrent radiation and chemotherapy.

for stage I-III unresectable esophageal SCC at 10 hospitals between 2010 and 2015. The patients included 126 men and 42 women. One hundred patients (59.5\%) received single-agent concurrent chemoradiotherapy and 68 (40.5\%) received dual-agent combination chemoradiotherapy. The average ages of the single-agent and double-agent groups were $62.75 \pm 7.8$ years and $58.32 \pm 9.17$ years, respectively $(P=0.037)$. The patient characteristics and treatment-related data are listed in Table 1.

\section{Toxicity}

Because poor follow up and some patients from day care unit, there were 112 patients with records of acute toxicities. In dual-agent group, the incidence of acute toxicity and the incidence of 3 and 4 grade toxicity were higher than single-agent group. The toxicity profiles are presented in Table 2.

\section{Survival}

The median PFS and OS of single-agent were 26 months [95\% confidence interval (CI), 12.6-39.4 months] and not reached. The median PFS and OS of double-agent groups were not reached. The estimate 5-year OS rates in the single-agent and double-agent groups were $62.7 \%$ and $78.2 \%$, respectively, and the corresponding 5 -year (PFS) rates were $40.9 \%$ and $52.5 \%$, respectively. Univariate analysis revealed no significant differences in OS and PFS between the single-agent and double-agent combination chemoradiotherapy groups $(P=0.161$ and 0.367 , respectively). The survival results are shown in Table 3, Fig. 1, and Fig. 2.

Multivariate analysis identified disease stage as the only factor associated with OS and PFS $(P=0.006$ and 0.003 , respectively). However, the use of a single-agent or double-agent regimen, sex, age, and radiotherapy dose had no significant effects on OS $(P=0.437,0.385,0.630$, 0.857 , respectively) or PFS $(P=0.5435,0.054,0.118$, 0.435 , respectively). The results of the multivariate analysis are listed in Table 4.

\section{Discussion}

Currently, radiotherapy is the major method of treatment for inoperable locally advanced esophageal cancers. However, a large review of 49 series involving $>8400$ patients who were treated primarily with radiation monotherapy reported 1-, 2-, and 5-year OS rates of 18\%, 8\%, and 6\%, respectively. The RTOG85-01 study was conducted to determine the role of radical concurrent chemoradiotherapy in the treatment of locally advanced esophageal cancer [5]. Since then, dual-agent concurrent chemoradiotherapy regimens have been widely used to treat unresectable esophageal cancer, and the National Comprehensive Cancer Network Guidelines recommend the use of 2 chemotherapeutic agents with concurrent radiotherapy [5, 7-11]. However, patients who received chemoradiotherapy had higher incidences of acute grade 3 (44\% vs. $25 \%)$ and grade 4 toxicity ( $20 \%$ vs. $3 \%$ ), compared with those who received radiation monotherapy [12]. Furthermore, reported chemoradiotherapy-related mortality rates ranged from $0 \%$ to $3 \%$ [13-16].

Single-dose concurrent chemoradiotherapy regimens, which yield equivalent effects with reduced toxicity, have been used to treat cervical cancers and head and neck cancers, and are recommended by the NCCN Guidelines 
Table 1 Patient characteristics and demographics

\begin{tabular}{|c|c|c|c|}
\hline Variable & $\begin{array}{l}\text { Single-agent } \\
\text { No. (\%) }\end{array}$ & $\begin{array}{l}\text { Two-agent } \\
\text { No. (\%) }\end{array}$ & $p$-value \\
\hline Total patients & 100 & 68 & \\
\hline Age at diagnosis (years) & & & 0.037 \\
\hline Mean \pm SD & $62.75 \pm 7.80$ & $58.32 \pm 9.17$ & \\
\hline (Range) & $(41-80)$ & $(36-79)$ & \\
\hline$\geq 65$ & $44(44)$ & & \\
\hline \multirow[t]{2}{*}{$<65$} & $56(56)$ & $21(31)$ & 0.087 \\
\hline & & $47(69)$ & \\
\hline Sex & & & 0.717 \\
\hline Male & $76(76)$ & $50(74)$ & \\
\hline Female & $24(24)$ & $18(26)$ & \\
\hline Location & & & 0.479 \\
\hline Cervical & $5(5)$ & $3(4)$ & \\
\hline Upper thoracic & $34(34)$ & $23(34)$ & \\
\hline Middle thoracic & $48(48)$ & $34(50)$ & \\
\hline Lower thoracic & $13(13)$ & $8(12)$ & \\
\hline ECOG PS & & & 0.069 \\
\hline 0 & $30(30)$ & $32(47)$ & \\
\hline 1 & $64(64)$ & $34(50)$ & \\
\hline 2 & $6(6)$ & 2(3) & \\
\hline Tumor stag & & & 0.896 \\
\hline $\mathrm{T} 1$ & $10(10)$ & $8(12)$ & \\
\hline $\mathrm{T} 2$ & $38(38)$ & $22(32)$ & \\
\hline T3 & $31(31)$ & $23(34)$ & \\
\hline T4 & $21(21)$ & $15(22)$ & \\
\hline Node stage & & & 0.360 \\
\hline NO & $56(56)$ & $31(46)$ & \\
\hline N1 & $24(24)$ & $18(26)$ & \\
\hline N2 & $20(20)$ & $19(28)$ & \\
\hline Clinical stage & & & 0.814 \\
\hline । & $24(24)$ & $15(22)$ & \\
\hline$\|$ & $44(44)$ & $28(41)$ & \\
\hline III & $32(32)$ & $25(37)$ & \\
\hline Radiotherapy dose (Gy) & & & 0.21 \\
\hline Mean \pm SD & $60.74 \pm 3.46$ & $61.97 \pm 3.28$ & \\
\hline (Range) & $(50-68)$ & $(54-70)$ & \\
\hline$>60$ & $26(26)$ & $26(38)$ & 0.092 \\
\hline$(50-60)$ & $74(74)$ & $42(62)$ & \\
\hline \multicolumn{4}{|l|}{ Chemotherapy regimens } \\
\hline $5-\mathrm{FU}$ & $23(23)$ & & \\
\hline Capecitabine & $10(10)$ & & \\
\hline S-1 & $37(37)$ & & \\
\hline Cisplatin & $25(25)$ & & \\
\hline Nadeplatin & $5(5)$ & & \\
\hline
\end{tabular}

Table 1 Patient characteristics and demographics (Continued)

\begin{tabular}{|c|c|c|c|}
\hline Variable & $\begin{array}{l}\text { Single-agent } \\
\text { №. (\%) }\end{array}$ & $\begin{array}{l}\text { Two-agent } \\
\text { No. (\%) }\end{array}$ & $p$-value \\
\hline Paclitaxel + Cisplatin & & $12(17)$ & \\
\hline Paclitaxel + carboplatin & & $9(13)$ & \\
\hline Docetaxel+ Cisplatin & & $6(9)$ & \\
\hline 5-FU+ Cisplatin & & $17(25)$ & \\
\hline 5-FU+ Oxaliplatin & & $5(7)$ & \\
\hline S-1 + Cisplatin & & $12(18)$ & \\
\hline Capecitabine+ Cisplatin & & $7(10)$ & \\
\hline
\end{tabular}

$[17,18]$. Single-dose concurrent chemoradiotherapy can also be used to treat esophageal cancer. Ji et al. reported that S1 single-agent concurrent chemoradiotherapy was well tolerated and effective in elderly patients with esophageal cancer and merited further investigation in phase I studies [19]. Cao et al. administered cisplatin concurrent chemoradiotherapy to 35 patients with cervical esophageal cancer. In that study, the overall 2-year local failure-free survival (LFFS), regional failure-free survival (RFFS), distant failure-free survival (DFFS), and OS rates were $68.3 \%, 80.4 \%, 67.7 \%$, and $46.1 \%$, respectively [20].

Our study results indicate that single therapy is not inferior to dual therapy for the treatment of unresectable esophageal SCC. In contrast, a previous retrospective study reported that single-agent concurrent chemoradiotherapy yielded better results with fewer side effects, compared with two-drug concurrent chemoradiotherapy [12]. In that study, 54 patients received tegafur suppositories (TF) and 86 received fluorouracil and cisplatin (PF) concurrently with external beam radiotherapy and neutron brachytherapy for localized advanced carcinoma of the esophagus. The analysis identified the chemotherapy regimen as the only factor associated with OS $(P=$ 0.025); factors such as sex, age, tumor length, tumor location, chemotherapy regimen, stage $\mathrm{T}$, stage $\mathrm{N}, \mathrm{AJCC}$ stage, and radiation dose were not significantly associated with OS. Furthermore, the study reported 5-year OS rates of $27.4 \%$ and $44.3 \%$ for the PF and TF chemotherapy regimens, respectively, and regimen-related severe, late complication rates of $9.3 \%(8 / 86)$ for the $\mathrm{PF}$ and $1.9 \%(1 / 54)$ for the TF regimens $(P=0.080)$.

Patients who received single-agent and double-agent combination chemoradiotherapy did not show significant differences in terms of OS and PFS, although double-agent treatment tended to prolong the survival time. The 5-year OS and PFS rates were $62.7 \%$ and $40.9 \%$, and $78.2 \%$ and $52.5 \%$ for the single-agent and double-agent chemotherapy regimens, respectively. The tendency of the latter to prolong survival in this study might be attributable to the younger age of patients in 
Table 2 Acute toxic effects $(n=112)$

\begin{tabular}{|c|c|c|c|c|}
\hline \multirow[t]{3}{*}{ Toxic effect } & \multicolumn{2}{|l|}{ All grades } & \multicolumn{2}{|l|}{ Grade 3 and 4} \\
\hline & Single-agent $(n=76)$ & Two-agent $(n=36)$ & Single-agent $(n=76)$ & Two-agent $(n=36)$ \\
\hline & No (\%) & No (\%) & No $(\%)$ & No (\%) \\
\hline Neutropenia & $49(64.5)$ & $30(83.3)$ & $26(34.2)$ & 19(52.7\%) \\
\hline Thrombocytopenia & 12(15.8) & $9(25.0)$ & $5(6.6)$ & $4(11.1)$ \\
\hline Anemia & $36(47.4)$ & $25(69.4)$ & $1(1.3)$ & $2(5.6)$ \\
\hline Nausea/vomiting & 14(18.4) & $11(30.1)$ & $1(1.3)$ & $1(2.8)$ \\
\hline Esophagitis & $51(67.1)$ & 28(77.8) & $6(7.9)$ & $3(8.3)$ \\
\hline Pneumonitis & $7(9.2)$ & $5(13.9)$ & $0(0)$ & $0(0)$ \\
\hline Liver function & 15(19.7) & $10(27.8)$ & $0(0)$ & $1(2.8)$ \\
\hline
\end{tabular}

the double-agent group. In a retrospective singleinstitution study, 239 esophageal cancers were treated with concurrent chemoradiotherapy. The 1, 2, and 3year OS rates were $60.5 \%, 44.4 \%$, and $34.6 \%$ for patients aged $>70$ years and $72.9 \%, 55.8 \%$, and $45.4 \%$ for those aged $<70$ years $(P=0.049)$ [21]. Hurmuzlu et al. reported univariate and multivariate analyses demonstrating an association between younger age and favorable OS $(P=0.017$ and $P=0.029$, respectively) [22]. In our study, patients $<65$ years of age tended to have a longer survival time, although they did not differ significantly from those aged $\geq 65$ years in terms of OS or PFS. The 5-year OS and PFS rates were $61.3 \%$ and $21.8 \%$ for those aged $\geq 65$ years and $74.9 \%$ and $55.7 \%$ for those aged < 65 years, respectively. Similar results were shown by Chen et al. who reported 5-year OS rates of $29.5 \%$ and

Table 3 Univariate analysis of prognostic factors related to overall survival (OS) and progression-free survival (PFS)

\begin{tabular}{lllll}
\hline Factor & 5 -year PFS (\%) & $p$ & 5 -year OS (\%) & $p$ \\
\hline Single or double & & & & \\
Single-agent & 40.9 & 0.367 & 62.7 & 0.161 \\
Double-agent & 52.5 & & 78.2 & \\
Stage & & & & 0.005 \\
I & 65.2 & 0.006 & 91.1 & \\
II & 42.7 & & 67.1 & \\
III & 32.8 & & 47.6 & \\
Sex & & 0.47 & & \\
Male & 42.2 & & 66.7 & \\
Female & 58.2 & & 75.6 & \\
Radiotherapy & & & & 0.735 \\
dose (Gy) & & & & \\
$>60$ & 43.8 & 0.287 & 65.8 & \\
$50-60$ & 50.6 & & 70.8 & \\
Age (years) & & & & \\
$\geq 65$ & 21.8 & 0.115 & 61.3 & \\
<65 & 55.7 & & 74.9 & \\
\hline
\end{tabular}

$23.3 \%$ for patients aged $\leq 60$ years and $>60$ years, respectively [23]. Furthermore, the RTOG85-01 study found that patients aged 60-69 years had a better prognosis than patients older than 69 years [5]. Because our study is a retrospective analysis, the result may be secondary to our study being underpowered.

Patients with relatively early disease can undergo resection; however, they cannot undergo operations due to surgical contraindications, such as poor cardiopulmonary function. Because the esophagus is a hollow organ, high radiotherapy dose cannot be administered, and chemotherapy adds to the effect of radiotherapy. In China, even patients with early disease would be treated with radiochemotherapy,

Other studies have identified age, TNM stage, radiotherapy dose, and chemotherapy regimen as prognostic factors affecting patients with esophageal cancer [5, 16, 24-26]. However, in the present study, only the TNM stage was found to affect prognosis and in multivariate analysis, only TNM stage remained a prognostic factor. Neither the univariate nor the multivariate analysis identified the chemotherapy regimen as a prognostic factor for esophageal cancer. Accordingly, double-agent regimens tend to prolong the survival of patients with esophageal cancer, but cannot affect prognosis.

Our study results yielded 5-year OS rates of $62.7 \%$ and $78.2 \%$ for the single-agent and double-agent groups respectively, and corresponding 5-year PFS rates of 40.9\% and52.5\%, respectively. These outcomes are superior to those of other studies [5, 16, 20, 24-27]. We attribute this difference to the exclusion of patients without any follow up data from our study. In China, especially the western region, population mobility is significant and patient compliance is poor; accordingly, many patients are not followed up after treatment completion, and hospital follow-up databases are flawed. Therefore, many patients were lost to follow-up after treatment, and most of the others died. We excluded patients without any follow up data, which led to increased 5-year survival rates. 


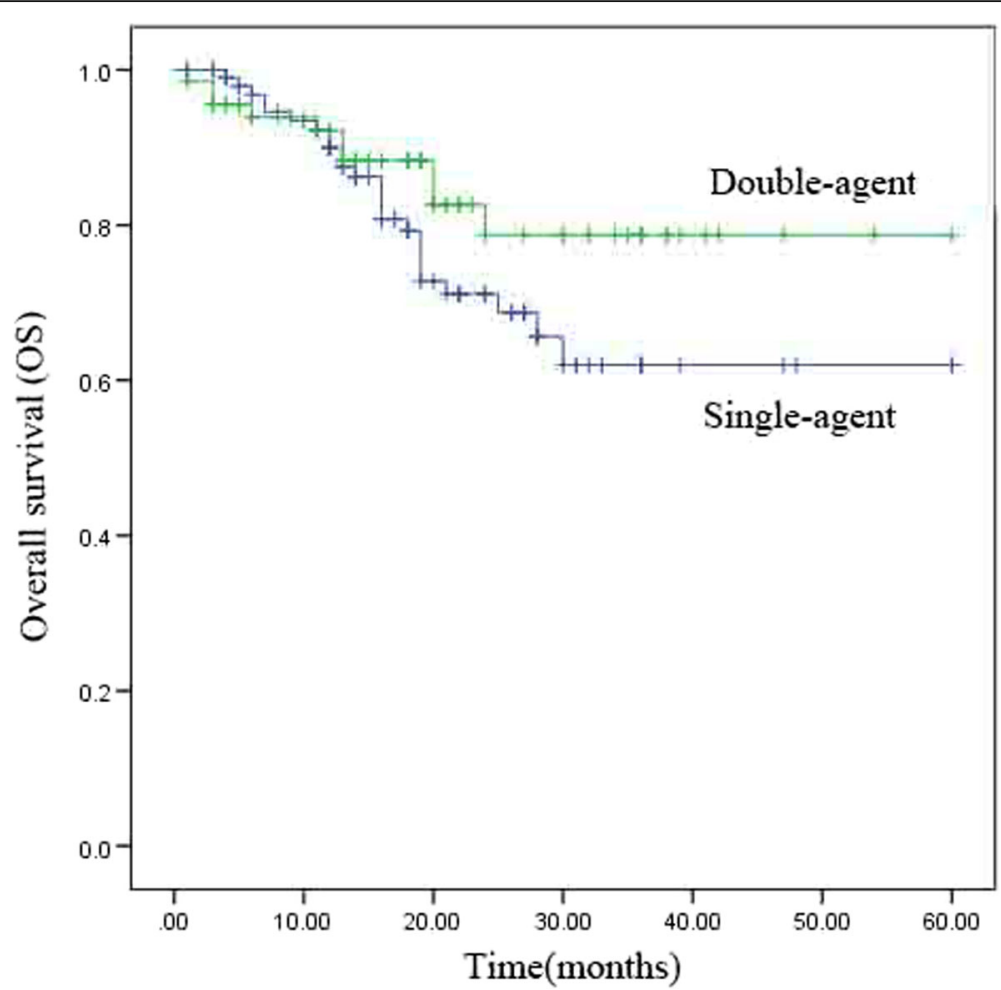

Fig. 1 Kaplan-Meier analysis of overall survival (OS)

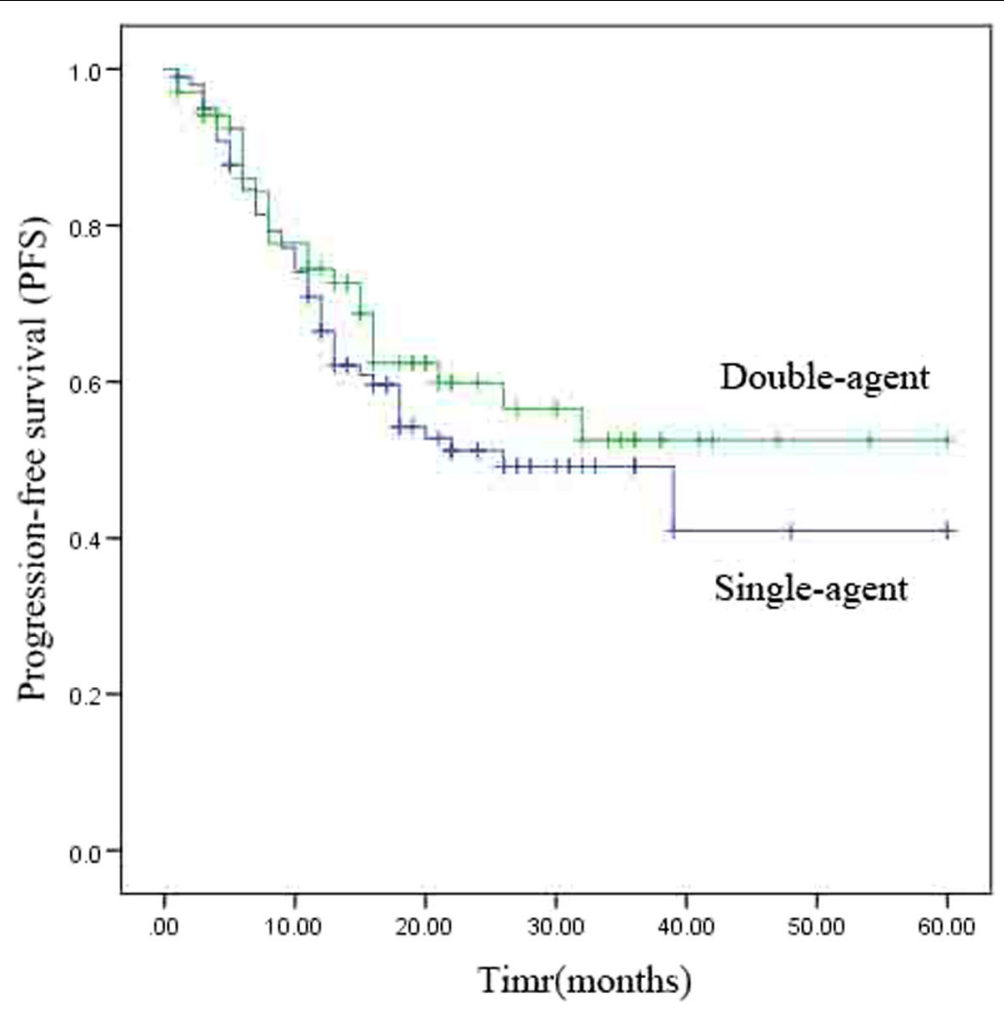

Fig. 2 Kaplan-Meier analysis of progression-free survival (PFS) 
Table 4 Multivariate analysis of prognostic factors related to overall survival (OS) and progression-free survival (PFS)

\begin{tabular}{lllll}
\hline Endpoint & Variable & HR & $95 \%$ Cl for HR & P \\
\hline PFS & Double agent & 0.821 & $0.5-1.347$ & 0.435 \\
Single agent & 1 & & \\
Stage II and III & 1.444 & $1.137-1.834$ & 0.003 \\
Stage I & 1 & & \\
Radiotherapy dose 50-60 Gy & 0.801 & $0.471-1.365$ & 0.415 \\
Radiotherapy dose >60Gy & 1 & & \\
Age $<65$ years & 0.679 & $0.418-1.103$ & 0.118 \\
Age $\geq 65$ years & 1 & & \\
Female & 0.541 & $0.290-1.01$ & 0.054 \\
Male & 1 & & \\
Double agent & 0.669 & $0.301-1.397$ & 0.437 \\
Single agent & 1 & & \\
Stagell and III & 1.607 & $1.146-2.253$ & 0.006 \\
Stage I & 1 & & \\
Radiotherapy dose 50-60 Gy & 0.935 & $0.452-1.937$ & 0.857 \\
Radiotherapy dose $>60 G y$ & 1 & & \\
Age $<65$ years & 0.845 & $0.425-1.678$ & 0.630 \\
Age $\geq 65$ years & 1 & & \\
Female & 0.692 & $0.301-1.589$ & 0.385 \\
Male & 1 & & \\
\hline
\end{tabular}

HR hazard ratio, $\mathrm{Cl}$ confidence interval

However, this exclusion had similar impacts on both the single-agent and double-agent groups.

Our study show that single-agent concurrent chemotherapy had less toxicity than a double-drug regimen in patients with esophageal cancer.. Multiple studies showed that single-agent concurrent chemotherapy was similarly efficacious and better tolerated compared with double-agent regimens for the treatment of head and neck cancer [28] and cervical cancer $[29,30]$. Another retrospective study reported less severe side effects with single-agent concurrent chemoradiotherapy compared with double-agent concurrent chemoradiotherapy [12].

\section{Conclusion}

A previous study reported that single-agent chemoradiation has better 5-year OS rates and less toxicity than double-agent therapy [12]. Our study shows that the 5-year PFS rate and OS rate of double-agent therapy was higher than that of single-agent concurrent chemoradiotherapy for patients with unresectable esophageal SCC; however, there were no significant differences in univariate analysis and multivariate analysis. The tendency of prolong survival might be attributable to the younger age of patients in the double-agent group. Single-agent concurrent chemotherapy had less toxicity than a double- drug regimen. Therefore, we hypothesize that single-agent therapy is not inferior to double-agent therapy. In the future, we aim to confirm our hypothesis through a prospective randomized study.

\section{Abbreviations}

AJCC: American joint committee on cancer; CT: Computed tomography; ECOG: Eastern United States tumor cooperation group; MRI: Magnetic resonance imaging; NCCN: National comprehensive cancer network; PET/ CT: Positron emission computed tomography; RTOG: Radiation therapy oncology group; SCC: Squamous cell carcinoma

\section{Acknowledgements}

Not applicable

\section{Funding}

This work was supported by the Mianyang Science and Technology Bureau (15-S01-3).

\section{Availability of data and materials}

The datasets supporting the conclusions of this article are included within the article.

\section{Authors' contributions}

Each author had participated sufficiently in the work to take public responsibility for appropriate portions of the content. XB Du designed this Study. J Li performed the statistical analysis. All authors helped to interpret the data. The manuscript was written by XB Du and J Li, all other authors helped and approved the final manuscript.

\section{Ethics approval and consent to participate}

The study was approved by the Medical Ethics Committee of Mianyang Central Hospital (approval no. S2016055). As clinical data were analyzed anonymously, ethics committee agrees that we were not required to obtain informed consent from the patients.

\section{Consent for publication}

Not applicable.

\section{Competing interests}

The authors declare that they have no competing interests.

\section{Publisher's Note}

Springer Nature remains neutral with regard to jurisdictional claims in published maps and institutional affiliations.

\section{Author details}

'Department of Oncology, Mian Yang Central Hospital, Mianyang 621000, People's Republic of China. ${ }^{2}$ Department of Oncology, West China Hospital of Sichuan University, Chengdu, China. ${ }^{3}$ Department of Oncology, Sichuan Cancer Hospital, Chengdu, China. ${ }^{4}$ Department of Oncology, Affiliated Hospital of North Sichuan Medical College, Nanchong, China. ${ }^{5}$ Department of Oncology, Lang Zhong People 's Hospital, Lang Zhong, China.

${ }^{6}$ Department of Oncology, Jiang You People s Hospital, Jiang You, China.

7Department of Oncology, Yan Ting County Cancer Hospital, Yan Ting, China. ${ }^{8}$ Department of Oncology, The Second Affiliated Hospital of Hainan Medical College, Haikou, China. 'Department of Oncology, Yibin Second People 's Hospital, Yibin, China. ${ }^{10}$ Department of Oncology, The Fourth People's Hospital of Zigong, Zigong, China.

Received: 3 August 2017 Accepted: 11 January 2018

Published online: 22 January 2018

\section{References}

1. Pennathur A, Gibson MK, Jobe BA, Luketich JD. Oesophageal carcinoma. Lancet. 2013;381:400-12.

2. Torre LA, Bray F, Siegel RL, Ferlay J, Lortet-Tieulent J, Jemal A. Global cancer statistics, 2012. CA Cancer J Clin. 2015 Mar;65(2):87-108.

3. Arnold M, Soerjomataram I, Ferlay J, et al. Global incidence of oesophageal cancer by histological subtype in 2012. Gut. 2015;64:381-7. 
4. Sagar PM, Gauperaa T, Sue-Ling H, et al. An audit of the treatment of cancer of the oesophagus. Gut. 1994;35:941-5.

5. Cooper JS, Guo MD, Herskovic A, et al. Chemoradiotherapy of locally advanced esophageal cancer: long-term follow-up of a prospective randomized trial (RTOG 85-01).Radiation therapy Oncology group. JAMA. 1999:281:1623-7.

6. Stage expert group of non surgical treatment of esophageal cancer in China, clinical staging criteria for non surgical treatment of esophageal cancer (Draft). Chin J Radiat Oncol. 2010;19(3):179-80.

7. Minsky BD, Pajak TF, Ginsberg RJ, et al. INT 0123 (radiation therapy Oncology group 94-05) phase III trial of combined-modality therapy for esophageal cancer: high-dose versus standard-dose radiation therapy. J Clin Oncol. 2002;20:1167-74.

8. Yamashita H, Haga A, Takenaka R, Kiritoshi T, Okuma K, Ohtomo K, et al. Efficacy and feasibility of ambulatory treatment-based monthly nedaplatin plus S-1 in definitive or salvage concurrent chemoradiotherapy for early, advanced, and relapsed esophageal cancer. Radiat Oncol. 2016 Jan 19;11:4.

9. Hihara J, Yoshida K, Hamai Y, Emi M, Yamaguchi Y, Wadasaki K. Phase I study of docetaxel (TXT) and 5-fluorouracil (5-FU) with concurrent radiotherapy in patients with advanced esophageal cancer. Anticancer Res. 2007 Jul-Aug:27(4C):2597-603.

10. Conroy T, Galais MP, Raoul JL, Bouché O, Gourgou-Bourgade S, Douillard JY, et al. Definitive chemoradiotherapy with FOLFOX versus fluorouracil and cisplatin in patients with oesophageal cancer (PRODIGE5/ACCORD17): final results of a randomised, phase 2/3 trial. Lancet Oncol. 2014 Mar;15(3):305-14.

11. National Comprehensive Cancer Network. Esophageal and Esophagogastric junction cancers, version 1. 2017. https://www.nccn.org/professionals/ physician_gls/f_guidelines.asp.

12. Ma K, Wang Q, Li T, Liu H, Liu B, Jia X, et al. A retrospective study of external beam radiation, neutron brachytherapy, and concurrent chemotherapy for patients with localized advanced carcinoma of the esophagus. Radiat Oncol. 2014;9:294.

13. Walsh TN, Noonan N, Hollywood D, et al. A comparison of multimodal therapy and surgery for esophageal adenocarcinoma. N Engl J Med. 1996; 335:462-7.

14. Burmeister BH, Smithers BM, Gebski V, et al. Surgery alone versus chemoradiotherapyfollowed by surgery for resectable cancer of the oesophagus: a randomised controlled phase III trial. Lancet Oncol. 2005;6: 659-68.

15. Herskovic A, Martz K, Al-Sarraf M, et al. Combined chemotherapy and radiotherapy compared with radiotherapy alone in patients with cancer of the esophagus. N Engl J Med. 1992;326:1593-8.

16. Stahl M, Stuschke M, Lehmann N, et al. Chemoradiation with and without surgery in patients with locally advanced squamous cell carcinoma of the esophagus. J Clin Oncol. 2005;23:2310-7.

17. National Comprehensive Cancer Network. Cervical cancer, Version1. 2017. https://www.nccn.org/professionals/physician_gls/pdf/head-and-neck.pdf.

18. National Comprehensive Cancer Network. Head-and-neck cancer, version 1. 2017. https://www.nccn.org/professionals/physician_gls/pdf/head-and-neck.pdf.

19. Ji Y, Qiu G, Sheng L, Sun X, Zheng Y, Chen M, Xianghui Du A. Phase I dose escalation study of S-1 with concurrent radiotherapy in elderly patients with esophageal cancer. J Thorac Dis. 2016:8(3):451-8.

20. Cao C, Luo J, Gao L, Xu G, Yi J, Huang X, et al. Definitive radiotherapy for cervical esophageal cancer. Head Neck. 2015;37(2):151-5.

21. Yamashita H, Takenaka R, Omori M, Imae T, Okuma K, Ohtomo K, et al. Involved-field radiotherapy (IFRT) versus elective nodal irradiation (ENI) in combination with concurrent chemotherapy for 239 esophageal cancers: a single institutional retrospective study. Radiat Oncol. 2015;10:171.

22. Hurmuzlu M, Øvrebø K, Monge OR, Smaaland R, Wentzel-Larsen T, et al. High-dose chemoradiotherapy followed by surgery versus surgery alone in esophageal cancer: a retrospective cohort study. World J Surg Oncol. 2010;8(1):46

23. Chen C-Z, Chen J-Z, Li D-R, Lin Z-X, Zhou M-Z, Li D-S, et al. Long-term outcomes and prognostic factors for patients with esophageal cancer following radiotherapy. World J Gastroenterol. 2013;19(10):1639-44.

24. Minsky BD, Neuberg D, Kelsen DP, et al. Final report of intergroup trial 0122 (ECOG PE-289, RTOG 90-12): phase II trial of neoadjuvant chemotherapy plus concurrent chemotherapy and high-dose radiation for squamous cell carcinoma of the esophagus. Int J Radiat Oncol Biol Phys. 1999;43:517-23.
25. Al-Sarraf M, Martz K, Herskovic A, et al. Progress report of combined chemoradiotherapy versus radiotherapy alone in patients with esophageal cancer: an intergroup study. J Clin Oncol. 1997;15:277-84.

26. Hurmuzlu M, Monge OR, Smaaland R, Viste A. High-dose definitive concomitant chemoradiotherapy in non-metastatic locally advanced esophageal cancer: toxicity and outcome. Dis Esophagus. 2010 Apr;23(3): 244-52.

27. Zhang P, Xi M, Zhao L, Qiu B, Liu H, Hu YH, Liu MZ. Clinical efficacy and failure pattern in patients with cervical esophageal cancer treated with definitive chemoradiotherapy. Radiother Oncol. 2015 Aug;116(2):257-61.

28. Rodriguez CP, Adelstein DJ, Rybicki LA, Savvides P, Saxton JP, Koyfman SA et al. Randomized phase III study of 2 cisplatin-based chemoradiation regimens in locally advanced head and neck squamous cell carcinoma: impact of changing disease epidemiology on contemporary trial design. Head Neck. 2015 Nov;37(11):1583-9.

29. Wang CC, Chou HH, Yang LY, Lin H, Liou WS, Tseng CW, et al. A randomized trial comparing concurrent chemoradiotherapy with singleagent cisplatin versus cisplatin plus gemcitabine in patients with advanced cervical cancer: an Asian gynecologic Oncology group study. Gynecol Oncol. 2015 Jun;137(3):462-7.

30. Dueñas-González A, Zarbá JJ, Patel F, Alcedo JC, Beslija S, Casanova L, et al. Phase III, open-label, randomized study comparing concurrent gemcitabine plus cisplatin and radiation followed by adjuvant gemcitabine and cisplatin versus concurrent cisplatin and radiation in patients with stage IIB to IVA carcinoma of the cervix. J Clin Oncol. 2011 May 1;29(13):1678-85.

\section{Submit your next manuscript to BioMed Central and we will help you at every step:}

- We accept pre-submission inquiries

- Our selector tool helps you to find the most relevant journal

- We provide round the clock customer support

- Convenient online submission

- Thorough peer review

- Inclusion in PubMed and all major indexing services

- Maximum visibility for your research

Submit your manuscript at www.biomedcentral.com/submit
Biomed Central 